\section{Fünf-Gräser-Tablette zeigt Langzeitwirk}

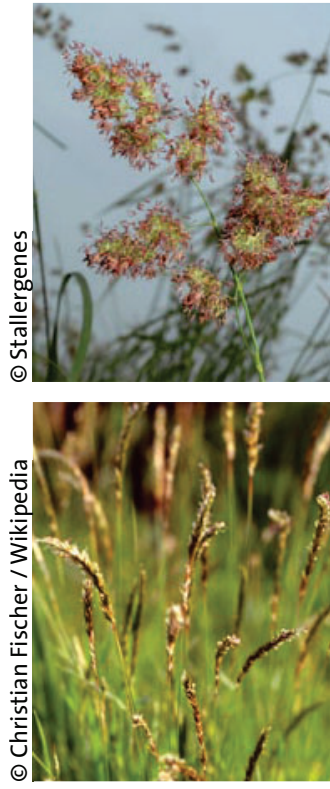

\section{Wiesenknäuel- gras (Dactylis glomerata)}

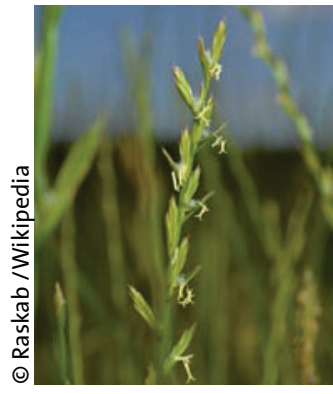

Gewöhnliches Ruchgras (Anthoxanthum odoratum)

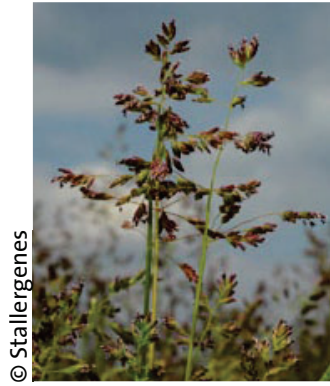

Deutsches Weidelgras (Lolium perenne)

Wiesenrispengras (Poa pratensis)

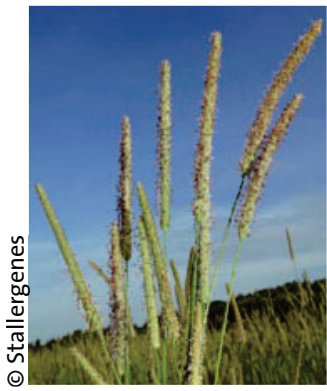

Wiesenlieschgras (Phleum pratense)

Jede Sublingualtablette Oralair ${ }^{\circledR}$ Gräser enthält zu gleichen Teilen Pollenextrakte von fünf Familienmitgliedern der Süßgräser ie spezifische Immuntherapie (SIT) ist inzwischen von einem empirischen zu einem evidenzbasierten Verfahren zur Behandlung der allergischen Rhinitis geworden. Wirksamkeit und Sicherheit konnten in den letzten Jahren in klinischen Studien klar belegt werden. Eine besonders gute Evidenzbasis besteht für die sublinguale Immuntherapie (SLIT) mit der 5-GräserTablette Oralair ${ }^{\circledR}$.

Die 5-Gräser-Tablette hat sich sowohl bei Erwachsenen als auch bei Kindern mit allergischer Rhinitis aufgrund einer Sensibilisierung gegen Gräserpollen als effektiv und sicher erwiesen. Durchgeführt wird die Therapie in drei aufeinanderfolgenden Jahren mit einem prä- bzw. co-saisonalen Therapieschema.

Wie Prof. Dr. Sabina Rak, Göteborg, Schweden, ausführte, hat die internationale Phase-III-Studie VO53.06 mit insgesamt 633 erwachsenen Patienten gezeigt, dass sich der Effekt im Laufe der dreijährigen Therapie im Vergleich zu Plazebo von Saison zu Saison verstärkt. Bereits in der ersten Saison war ein signifikanter Unterschied im kombinierten SymptomMedikations-Score zu beobachten: Der Scorewert war bei viermonatiger präsaisonaler und co-saisonaler Therapie um $18 \%(\mathrm{p}<0,0008)$ geringer. Im zweiten Jahr stieg die relative Symptomreduktion auf $32 \%(\mathrm{p}<0,0001)$, im dritten Jahr auf $35 \%(\mathrm{p}<0,0001)$.

Eine aktuelle Auswertung des ersten therapiefreien Jahres - der vierten Saison - ergab, dass der Effekt auch nach dem Ende der dreijährigen Therapie anhält. Der Symptom-MedikationsScore war um $23 \%(p=0,0112)$ geringer als in der Plazebogruppe.

\section{Dr. Angelika Bischoff}

Pressekonferenz und Symposium „Grass and mites immunotherapy tablets: consolidating of the new therapeutic class" im Rahmen des 30. Kongresses der European Academy of Allergy and Clinical Immunology. Istanbul, Türkei, 12. Juni 2011. Veranstalter: Stallergenes, Antony, Frankreich

\section{Fliegender Wechsel}

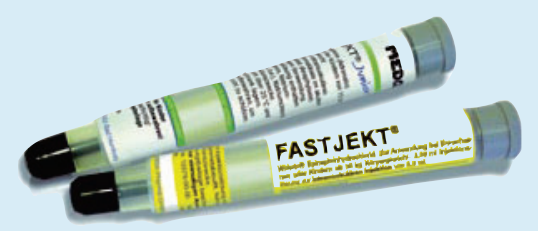

Seit Juni dieses Jahres hat MEDA Pharma in Bad Homburg den exklusiven Vertrieb für die Adrenalin-Autoinjektoren Fastjekt ${ }^{\circledR}$ und Fastjekt ${ }^{\circledR}$ Junior in Deutschland übernommen. Das Präparat Fastjekt ${ }^{\circledR}$ zur Notfallbehandlung von Anaphylaxien für Erwachsene und Kinder ab einem Körpergewicht von $30 \mathrm{~kg}$ enthält o,3 mg Adrenalin, Fastjekt ${ }^{\oplus}$ Junior für Kinder von 15 bis $30 \mathrm{~kg}$ Körpergewicht o,15 mg Adrenalin. Beide werden von der US-amerikanischen Mylan-Tochter Dey Pharma hergestellt und waren in Deutschland bislang über das Unternehmen Allergopharma erhältlich. Ungeachtet des Vertriebsübergangs wird das Präparat in gewohnter Qualität und ohne Verzögerung lieferbar sein. In Österreich und der Schweiz wird es übrigens unter dem Namen EpiPen ${ }^{\circledast}$ vermarktet. red

Nach Informationen von MEDA Pharma, Bad Homburg

\section{Fragebogen bereitet vor}

Unter www. whiar.org/Intl/ QuestionnaireGER steht $a b$ sofort ein Fragebogen zu allergischer Rhinitis und Asthma zur Verfügung, den

Patienten vor dem Arztbesuch beantworten und zur Vorbereitung ihres Gesprächs mit in die Praxis nehmen können. Der Fragebogen wird direkt am Bildschirm ausgefüllt. Patienten werden durch verschiedene Fragen rund um Art und Häufigkeit verschiedener Symptome geführt und haben am Ende die Möglichkeit, sich ihre Ergebnisse auszudrucken oder per E-Mail zuschicken zu lassen. Der Fragebogen wurde entwickelt von der ARIA-Initiative (Allergic Rhinitis and its Impact on Asthma). Diese Initiative will weltweit über die Zusammenhänge und die Behandlung von allergischer Rhinitis und Asthma aufklären.

Nach Informationen von Merckle Recordati, UIm 